Working Paper 99-87

Statistics and Econometrics Series 35

December 1999
Departamento de Estadística y Econometría Universidad Carlos III de Madrid Calle Madrid, 126 28903 Getafe (Spain)

Fax (34-91) 624-9849

\title{
LIMITING DISCOUNTED-COST CONTROL OF PARTIALLY OBSERVABLE STOCHASTIC SYSTEMS.
}

Onésimo Hernández-Lerma and Rosario Romera.*

\section{Abstract}

This paper presents two main results on partially observable (PO) stochastic systems. In the first one, we consider a general PO system

$$
\mathrm{x}_{\mathrm{t}+1}=\mathrm{F}\left(\mathrm{x}_{\mathrm{t}}, \mathrm{a}_{\mathrm{t}}, \xi_{\mathrm{t}}\right), \quad \mathrm{y}_{\mathrm{t}}=\mathrm{G}\left(\mathrm{x}_{\mathrm{t}}, \eta_{\mathrm{j}}\right) \quad(\mathrm{t}=0,1, \ldots)
$$

on Borel spaces, with possibly unbounded cost-per-stage functions, and give conditions for the existence of $\alpha$-discount optimal control policies $(0<\alpha<1)$.

In the second result we specialize $\left({ }^{*}\right)$ to additive-noise systems

$$
x_{t+1}=F_{n}\left(x_{t}, a_{t}\right)+\xi_{t}, \quad y_{t}=G_{n}\left(x_{t}\right)+\eta_{t} \quad(t=0,1, \ldots)
$$

in Euclidean spaces, with $F_{n}(x, a)$ and $G_{n}(x)$ converging pointwise to functions $F_{\infty}(x, a)$ and $G_{\infty}(x)$, respectively, and give conditions for the limiting PO model

$$
\mathrm{x}_{\mathrm{t}+1}=\mathrm{F}_{\infty}\left(\mathrm{x}_{\mathrm{t}}, \mathrm{a}_{\mathrm{t}}\right)+\xi_{\mathrm{t}} \quad \mathrm{y}_{\mathrm{t}}=\mathrm{G}_{\infty}\left(\mathrm{x}_{\mathrm{t}}\right)+\eta_{\mathrm{t}}
$$

to have an $\alpha$-discount optimal policy.

Keywords: Partially observable control systems; partially observable Markov control processes; hidden Markov models; discounted cost criterion.

*Hernández-Lerma, Departamento de Matemáticas, CINVESTAV-IPN, A. Postal 14740, México D.F. 07000, México. Tfno: (52-5)7473871. Fax: (52-5)747-3876, email: ohernand@math.cinvestav.mx; Romera, Universidad Carlos III de Madrid, Departamento de Estadística y Econometría, C/ Madrid, 126-128, Spain. Tfno: (3491)6249864; fax: (34-91)6249849; e-mail. Imrromera@est-econ.uc3m.es; Research partially supported by CONACYT (México) Grant 32299-E for OHL, and DGES (Spain) Grant PB96-0111 for R.R. 


\section{Introduction}

In this paper we consider a nonlinear, time-varying, partially observable (PO) stochastic control system with state process $\left\{x_{t}\right\}$ evolving according to the equation

$$
x_{t+1}=F_{t}\left(x_{t}, a_{t}\right)+\xi_{t}, \quad t \in \mathbb{N},
$$

where $\mathbb{N}:=\{0,1, \ldots\}$, and observations $\left\{y_{t}\right\}$ of the form

$$
y_{t}=G_{t}\left(x_{t}\right)+\eta_{t}, \quad t \in \mathbb{N} .
$$

Assuming that the functions $F_{t}$ and $G_{t}$ converge pointwise to functions $F_{\infty}$ and $G_{\infty}$, that is, as $t \rightarrow \infty$

$$
F_{t}(x, a) \rightarrow F_{\infty}(x, a) \text { and } \quad G_{t}(x) \rightarrow G_{\infty}(x)
$$

for all $(x, a)$ and $x$, respectively, we investigate the existence of optimal control policies for the limiting $P O$ system

$$
x_{t+1}=F_{\infty}\left(x_{t}, a_{t}\right)+\xi_{t}, \quad y_{t}=G_{\infty}\left(x_{t}\right)+\eta_{t},
$$

when the optimality criterion is the $\alpha$-discounted cost $(0<\alpha<1)$.

In fact, we present two main, different, results. In the first one, we consider a general PO system

$$
x_{t+1}=F\left(x_{t}, a_{t}, \xi_{t}\right), \quad y_{t}=G\left(x_{t}, \eta_{t}\right)
$$

in which the state space $\mathrm{X}$ and the observation set $\mathrm{Y}$ are Borel spaces (that is, Borel subsets of complete and separable metric spaces). Similarly, the state and observation disturbances $\xi_{t}$ and $\eta_{t}$ take values in Borel spaces $S$ and $S^{\prime}$, respectively, whereas the control actions $a_{t}$ are taken from a compact metric space $A$. In this setting, we give conditions for the existence of $\alpha$-discount optimal policies, allowing the cost-per-stage to be possibly unbounded. (See Theorem 2.4.)

In the second main result (Theorem 2.6), we consider the additive-noise case (1.1), (1.2) and the limiting system (1.4), on the spaces $\mathrm{X}=S=\mathbb{R}^{d_{1}}$ and $\mathrm{Y}=S^{\prime}=\mathbb{R}^{d_{2}}$. Assuming (1.3), we give conditions ensuring the existence of a control policy for (1.4).

To prove these results we begin by writing (1.5) as a PO Markov control (or decision) process, also known as a controlled "hidden Markov model" [5]. In other words, we work with a general state transition law and a general observation kernel, 
as in (2.10) and (2.11), respectively, which can be specialized in the obvious manner to (1.5), say. (See (2.12) and (2.13).) The formulation (2.10), (2.11) has, of course, technical advantages, but what is even more important is that it includes a class of models larger than (1.5). Namely, there are many applications in control of queues, fisheries, learning processes, and others (see [3, 5, 6, 10, 13, 16, 17]) described by "stochastic kernels" as in (2.10) and (2.11), on possibly finite or countable spaces, rather than by a "difference equation" model such as (1.5). Moreover, using (2.10), (2.11), our Theorem 2.6, when (1.3) holds, is easily related to results on either the approximation or the adaptive control of PO systems, or even for the completely observable $(\mathrm{CO})$ case which results when $y_{t}=x_{t}$ for all time index $t$; see $[3,4,6,7$, $10,11,12,14,18]$. Similarly, in the non-controlled case (namely, when the control space A is a one-point set, say), our results on (1.1)-(1.4) can be seen as stating the convergence of filtering models - see Lemma 4.1 .

Our approach is somewhat related to the CO case considered in [12], but the technical requirements are quite different. This is due to the fact that the analysis of (1.5) requires to introduce an equivalent $\mathrm{CO}$ system with values in a set of probability measures (see (2.5)-(2.7)). Thus, for instance, some "pointwise" statements in [12], in our present setting turn out to be statements on the convergence of measures in some suitable sense. (See, in particular, the comments in $\S 5$ below.)

The remainder of the paper is organized as follows. In $\S 2$ we state our assumptions, the control problems we are concerned with, and our main results, Theorem 2.4 and Theorem 2.6. Their proofs are presented in $\S 3$ and $\S 4$, respectively. We conclude in $\S 5$ with some general comments.

\section{The general PO system}

We begin with the following remark on the terminology and notation we shall use, and then proceed to state the optimal control problem we are concerned with.

Remark 2.1. (a) Given a Borel space $\mathrm{X}$, we denote by $\mathcal{B}(\mathrm{X})$ its Borel $\sigma$-algebra, and by $\mathbb{P}(\mathrm{X})$ the family of probability measures on $\mathrm{X}$, endowed with the usual weak topology $\sigma\left(\mathbb{P}(\mathrm{X}), C_{b}(\mathrm{X})\right)$, where $C_{b}(\mathrm{X})$ stands for the Banach space of continuous bounded functions $u$ on $\mathrm{X}$ with the sup norm $\|u\|:=\sup _{x}|u(x)|$. Thus, a sequence $\left\{\mu_{k}\right\}$ in $\mathbb{P}(\mathrm{X})$ is said to converge weakly to $\mu$ if

$$
\int_{\mathrm{X}} u d \mu_{k} \rightarrow \int_{\mathrm{X}} u d \mu \quad \forall u \in C_{b}(\mathrm{X})
$$


As $\mathrm{X}$ is a Borel space, so is $\mathbb{P}(\mathrm{X})$. (See $[1,2,19]$, for instance.)

(b) Let $\mathrm{X}$ and $\mathrm{Y}$ be Borel spaces. A measurable function $q: \mathrm{Y} \rightarrow \mathbb{P}(\mathrm{X})$ is called a stochastic kernel on $\mathrm{X}$ given $\mathrm{Y}$, and we denote by $\mathbb{P}(\mathrm{X} \mid \mathrm{Y})$ the family of all those stochastic kernels. Equivalently, $q(d x \mid y)$ is in $\mathbb{P}(\mathrm{X} \mid \mathrm{Y})$ if $q(\cdot \mid y)$ is a probability measure on $\mathrm{X}$ for each fixed $y \in \mathrm{Y}$, and $q(B \mid \cdot)$ is a measurable function on $\mathrm{Y}$ for each fixed $B \in \mathcal{B}(\mathrm{X})$. If $\mathrm{X}=\mathrm{Y}$, then a stochastic kernel is called a Markov transition probability.

Throughout the following we suppose:

Assumption 2.2. All the stochastic processes considered below are defined on an underling probability space $(\Omega, \mathcal{F}, P)$. In addition:

(a) The sate space $\mathrm{X}$, the observation set $\mathrm{Y}$, and the disturbance spaces $S$ and $S^{\prime}$ are all Borel spaces.

(b) The control (or action) set $A$ is a compact metric space.

(c) The state and observation disturbances $\xi_{t}$ and $\eta_{t}, t \in \mathbb{N}$, form independent sequences of i.i.d. (independent and identically distributed) random variables with values in $S$ and $S^{\prime}$, respectively. These sequences are also independent of the initial state $x_{0}$. We denote by $\mu \in \mathbb{P}(S)$ and $\nu \in \mathbb{P}\left(S^{\prime}\right)$ the common distributions of $\xi_{t}$ and $\eta_{t}$, respectively.

(d) The functions $F(x, a, s)$ and $G\left(x, s^{\prime}\right)$ in (1.5) are continuous.

(e) The cost-per-stage function $c: \mathrm{X} \times A \rightarrow \mathbb{R}$ is nonnegative and lower semicontinuous.

(f) There exists a constant $C$ and a continuous function $w \geq 1$ on $\mathrm{X}$ such that $c(x, a) \leq C w(x)$ for all $x \in \mathrm{X}$ and $a \in A$.

The PO control problem. Let $\mathcal{Y}_{t}:=\sigma\left(y_{0}, \ldots, y_{t}\right)$ be the $\sigma$-algebra generated by the observations up to time $t$. By an admissible control policy (or simply a policy) we mean a sequence $\pi=\left\{a_{t}\right\}$ of $A$-valued random variables such that $a_{t}$ is $\mathcal{Y}_{t}$-measurable for each $t \in \mathbb{N}$. We shall denote by $\Pi$ the set of all such policies. 
Let $\alpha \in(0,1)$ be a fixed "discount factor". For each policy $\pi \in \Pi$ and initial distribution $\varphi \in \mathbb{P}(\mathrm{X})$ ( that is, $\varphi$ is the a priori distribution of $x_{0}$ ), the corresponding $\alpha$-discounted cost is defined as

$$
V(\pi, \varphi):=\sum_{t=0}^{\infty} \alpha^{t} E_{\varphi}^{\pi}\left[c\left(x_{t}, a_{t}\right)\right]
$$

where $E_{\varphi}^{\pi}$ denotes the expectation operator with respect to the probability measure $P_{\varphi}^{\pi}$ induced by $\pi$ and $\varphi$. Let

$$
V^{*}(\varphi):=\inf _{\pi} V(\pi, \varphi), \quad \text { for } \quad \varphi \in \mathbb{P}(\mathrm{X})
$$

be the optimal $\alpha$-discounted cost. The PO optimal control problem is then to find an optimal policy $\pi^{*}$, that is, a policy such that

$$
V\left(\pi^{*}, \varphi\right)=V^{*}(\varphi) \quad \forall \varphi \in \mathbb{P}(\mathrm{X}) .
$$

The CO control problem. To study the PO control problem we shall follow the standard procedure, in which the $\mathrm{PO}$ problem is transformed into a completely observable (CO) problem using the filtering process $\left\{\varphi_{t}\right\}$ in $\mathbb{P}(\mathrm{X})$ defined as follows: For each policy $\pi \in \Pi$ and initial distribution $\varphi \in \mathbb{P}(\mathrm{X})$,

$$
\begin{aligned}
& \varphi_{0}(B):=P_{\varphi}^{\pi}\left(x_{0} \in B\right)=\varphi(B), \\
& \varphi_{t}(B):=P_{\varphi}^{\pi}\left(x_{t} \in B \mid \mathcal{Y}_{t}\right) \text { for } \quad t \geq 1,
\end{aligned}
$$

which are defined for all $B$ in $\mathcal{B}(\mathrm{X})$. The filtering process depends, of course, on the policy $\pi$ and the initial distribution $\varphi$, and so, strictly speaking, we should write $\varphi_{t}$ as, say, $\varphi_{t, \varphi}^{\pi}$. However, we shall use the simpler notation in (2.5) and (2.6) unless we need to remark which $\pi$ and $\varphi$ are being used.

To continue with the description of the PO problem, we use the well-known fact (see, for instance $[1,5,21,22]$ and Example 2.5 below) that there exists a measurable function $H: \mathbb{P}(\mathrm{X}) \times A \times \mathrm{Y} \rightarrow \mathbb{P}(\mathrm{X})$ such that (2.6) can be written as

$$
\varphi_{t+1}=H\left(\varphi_{t}, a_{t}, y_{t+1}\right) \quad \forall t \in \mathbb{N},
$$

with initial condition (2.5). (Note that, by the Remark $2.1(\mathrm{~b}), H$ is a stochastic kernel on $\mathbb{P}(\mathrm{X})$ given $\mathbb{P}(\mathrm{X}) \times A \times \mathrm{Y}$.) Moreover, using the notation

$$
\widehat{c}(\varphi, a):=\int_{\mathrm{X}} c(x, a) \varphi(d x) \text { for } \varphi \in \mathbb{P}(\mathrm{X}), a \in A
$$


we can rewrite the $\alpha$-discounted cost in (2.2) as

$$
V(\pi, \varphi)=\sum_{t=0}^{\infty} \alpha^{t} E_{\varphi}^{\pi}\left[\widehat{c}\left(\varphi_{t}, a_{t}\right)\right] .
$$

Finally, the CO problem is to minimize (2.9) over all $\pi \in \Pi$, subject to (2.5) and (2.6), and this problem is equivalent to the original $\mathrm{PO}$ one in the sense that an optimal policy for $\mathrm{CO}$ is optimal for $\mathrm{PO}$.

Solution of the CO problem. To state our first main result in this section, we need some notation. Let $P \in \mathbb{P}(\mathrm{X} \mid \mathrm{X} \times A)$ and $Q \in \mathbb{P}(\mathrm{Y} \mid \mathrm{X})$ be state transition law and the observation kernel corresponding to (1.5), that is,

$$
P(B \mid x, a):=\operatorname{Prob}\left(x_{t+1} \in B \mid x_{t}=x, a_{t}=a\right)
$$

and

$$
Q(C \mid x):=\operatorname{Prob}\left(y_{t} \in C \mid x_{t}=x\right)
$$

for each $B \in \mathcal{B}(\mathrm{X}), C \in \mathcal{B}(\mathrm{Y}), x \in \mathrm{X}, a \in A$, and $t \in \mathbb{N}$. More explicitly, in view of (1.5) and Assumption 2.2(c), we have that

$$
P(B \mid x, a)=\int_{S} I_{B}[F(x, a, s)] \mu(d s)
$$

and

$$
Q(C \mid x)=\int_{S^{\prime}} I_{C}\left[G\left(x, s^{\prime}\right)\right] \nu\left(d s^{\prime}\right),
$$

where $I_{B}$ denotes the indicator function of a set $B$. Moreover, for each $C \in \mathcal{B}(\mathrm{Y}), \varphi \in$ $\mathbb{P}(\mathrm{X})$, and $a \in A$, consider the stochastic kernel

$$
\widehat{q}(C \mid \varphi, a):=\operatorname{Prob}\left(y_{t+1} \in C \mid \varphi_{t}=\varphi, a_{t}=a\right),
$$

which using (2.10)-(2.13) can be written as

$$
\begin{aligned}
\widehat{q}(C \mid \varphi, a) & =\int_{\mathrm{X}} \int_{\mathrm{X}} Q\left(C \mid x^{\prime}\right) P\left(d x^{\prime} \mid x, a\right) \varphi(d x) \\
& =\int_{\mathrm{X}} \int_{S} \int_{S^{\prime}} I_{C}\left[G\left(F(x, a, s), s^{\prime}\right)\right] \nu\left(d s^{\prime}\right) \mu(d s) \varphi(d x)
\end{aligned}
$$


Finally, for each $D \in \mathcal{B}(\mathbb{P}(\mathrm{X})), \varphi \in \mathbb{P}(\mathrm{X}), a \in A$, and $t \in \mathbb{N}$, let

$$
\widehat{P}(D \mid \varphi, a):=\operatorname{Prob}\left(\varphi_{t+1} \in D \mid \varphi_{t}=\varphi, a_{t}=a\right)
$$

be the transition law of the filtering process (2.7), which we can also write as

$$
\widehat{P}(D \mid \varphi, a)=\int_{Y} I_{D}[H(\varphi, a, y)] \widehat{q}(d y \mid \varphi, a) .
$$

Assumption 2.3. Let $H$ and $w \geq 1$ be as in (2.7) and Assumption 2.2(f), respectively, and define $\widehat{w}: \mathbb{P}(\mathrm{X}) \rightarrow \mathbb{R}$ as $\widehat{w}(\varphi):=\int_{\mathrm{X}} w(x) \varphi(d x)$.

(a) $H$ is continuous;

(b) There is a number $1 \leq \beta<1 / \alpha$ such that

$$
\int_{\mathbb{P}(X)} \widehat{w}\left(\varphi^{\prime}\right) \widehat{P}\left(d \varphi^{\prime} \mid \varphi, a\right) \leq \beta \widehat{w}(\varphi) \quad \forall \varphi \in \mathbb{P}(\mathrm{X}), \quad a \in A .
$$

Observe that the property " $w \geq 1$ " of $w$ is inherited by $\widehat{w}$, because

$$
\widehat{w}(\varphi):=\int_{\mathrm{X}} w d \varphi \geq \varphi(\mathrm{X})=1 \quad \forall \varphi \in \mathbb{P}(\mathrm{X})
$$

We shall denote by $\mathbb{B}_{w}(\mathbb{P}(X))$ the (vector) space of all real-valued measurable functions $u$ on $\mathbb{P}(\mathrm{X})$ such that

$$
\|u\|_{w}:=\sup _{\varphi}|u(\varphi)| / \widehat{w}(\varphi)<\infty
$$

We can now state our first optimality result as follows.

Theorem 2.4. If Assumptions 2.2 and 2.3 are satisfied, then:

(a) The optimal cost function $V^{*}(\varphi):=\inf _{\pi} V(\pi, \varphi)$, with $V(\pi, \varphi)$ as in (2.9), is the unique solution in $\mathbb{B}_{w}(\mathbb{P}(\mathrm{X}))$ of the Bellman (or Dynamic Programming) equation

$$
V^{*}(\varphi)=\min _{a \in A}\left[\widehat{c}(\varphi, a)+\alpha \int_{\mathbb{P}(\mathrm{X})} V^{*}\left(\varphi^{\prime}\right) \widehat{P}\left(d \varphi^{\prime} \mid \varphi, a\right)\right]
$$

for all $\varphi \in \mathbb{P}(\mathrm{X})$. Moreover, 
(b) $V^{*}$ is l.s.c., and

(c) there exists a measurable function $f^{*}: \mathbb{P}(\mathrm{X}) \rightarrow A$ that attains the minimum in (2.20), i.e., for all $\varphi \in \mathbb{P}(\mathrm{X})$

$$
V^{*}(\varphi)=\widehat{c}\left(\varphi, f^{*}(\varphi)\right)+\alpha \int_{\mathbf{P}(\mathrm{X})} V^{*}\left(\varphi^{\prime}\right) \widehat{P}\left(d \varphi^{\prime} \mid \varphi, f^{*}(\varphi)\right),
$$

and $f^{*}$ determines optimal control policy $\pi^{*}=\left\{a_{t}^{*}\right\}$ given by

$$
a_{t}^{*}:=f^{*}\left(\varphi_{t}\right) \quad \forall t \in \mathbb{N},
$$

where $\left\{\varphi_{t}\right\}$ is the filtering process.

Theorem 2.4, which is proved in $\S 3$, is essentially standard except for the fact that we are allowing a general PO system (1.5) and a possibly unbounded cost-perstage $c(x, a)$, as in Assumption 2.2(e), (f). To the best of our knowledge, the only case studied in the literature in which $c(x, a)$ is unbounded is for the so-called $L Q G$ (Linear-Quadratic-Gaussian) PO systems. Furthermore, the existence of the "filtering function" $H$ in (2.7) depends only of the state transition law and the observation kernel in (2.10) and (2.11), not on the particular PO model (1.5). This means, in other words, that Theorem 2.4 is valid for general PO systems on Borel spaces, and so, in particular, it includes systems on countable spaces, which are very common in applications; see $[3,5,6,10,13,16,20]$.

We conclude this section with an example on an additive-noise system, which serves several purposes: it illustrates the concepts introduced above; it is an "introduction" to study the limiting system (1.4); and it gives conditions under which Assumption 2.3(a) is satisfied.

Example 2.5 . Consider the PO additive-noise system

$$
x_{t+1}=F\left(x_{t}, a_{t}\right)+\xi_{t}, \quad y_{t}=G\left(x_{t}\right)+\eta_{t}, \quad t \in \mathbb{N}
$$

with $\mathrm{X}=S=\mathbb{R}^{d_{1}}, \mathrm{Y}=S^{\prime}=\mathbb{R}^{d_{2}}$, and $A$ compact metric; see Assumptions 2.2(a), (b). In addition, the disturbances $\left\{\xi_{t}\right\}$ and $\left\{\eta_{t}\right\}$ are as in Assumption 2.2(c), except that now we also suppose: 
Hypothesis $\mathbf{A}$. The noise distributions $\mu$ and $\nu$ are absolutely continuous, say

$$
\mu(d s)=g_{\xi}(s) \lambda_{1}(d s) \quad \text { and } \quad \nu\left(d s^{\prime}\right)=g_{\eta}\left(s^{\prime}\right) \lambda_{2}\left(d s^{\prime}\right)
$$

where $\lambda_{i}(i=1,2)$ denotes the Lebesgue measure on $\mathbb{R}^{d_{i}}$, and, moreover, $g_{\xi}$ and $g_{\eta}$ are continuous bounded density functions.

In this case, the state transition law in (2.10), (2.12) becomes

$$
P(B \mid x, a)=\int_{B} g_{\xi}(s-F(x, a)) \lambda_{1}(d s)
$$

and, similarly, the observation kernel in (2.11), (2.13) becomes

$$
Q(C \mid x)=\int_{C} g_{\eta}\left(s^{\prime}-G(x)\right) \lambda_{2}\left(d s^{\prime}\right)
$$

On the other hand, as is well-known $[3,7,11,20,21]$, the filtering function $H$ in $(2.7)$ is of the form

$$
H(\varphi, a, y)(B)=\sigma(\varphi, a, y)(B) / \sigma(\varphi, a, y)(\mathrm{X}) \quad \forall B \in \mathcal{B}(\mathrm{X})
$$

with

$$
\begin{aligned}
\sigma(\varphi, a, y)(B) & =\int_{B} g_{\eta}\left(y-G\left(x^{\prime}\right)\right) \int_{\mathrm{X}} P\left(d x^{\prime} \mid x, a\right) \varphi(d x) \\
& =\int_{\mathrm{X}}\left[\int_{B} g_{\eta}\left(y-G\left(x^{\prime}\right)\right) P\left(d x^{\prime} \mid x, a\right)\right] \varphi(d x) \\
& =\int_{\mathrm{X}}\left[\int_{B} g_{\eta}\left(y-G\left(x^{\prime}\right)\right) g_{\xi}\left(x^{\prime}-F(x, a)\right) \lambda_{1}\left(d x^{\prime}\right)\right] \varphi(d x),
\end{aligned}
$$

by (2.24).

On the other hand, Assumption 2.2(d) reduces to:

Hypothesis B. The functions $F: \mathrm{X} \times A \rightarrow \mathrm{X}$ and $G: \mathrm{X} \rightarrow \mathrm{Y}$ are continuous.

We can then see from the general Lemma 3.2, below, that $H$ satisfies Assumption 2.3(a). Indeed, let $\left(\varphi^{k}, a^{k}, y^{k}\right)$ be a sequence in $\mathbb{P}(\mathrm{X}) \times A \times \mathrm{Y}$ that converges to $(\varphi, a, y)$. Choose an arbitrary function $u$ in $C_{b}(\mathrm{X})$, and define

$$
v^{k}(x):=\int_{\mathrm{X}} u\left(x^{\prime}\right) g_{\eta}\left(y^{k}-G\left(x^{\prime}\right)\right) g_{\xi}\left(x^{\prime}-F\left(x, a^{k}\right)\right) \lambda_{1}\left(d x^{\prime}\right),
$$




$$
v(x):=\int_{\mathrm{X}} u\left(x^{\prime}\right) g_{\eta}\left(y-G\left(x^{\prime}\right)\right) g_{\xi}\left(x^{\prime}-F(x, a)\right) \lambda_{1}\left(d x^{\prime}\right) .
$$

Observe that, by Hypothesis $\mathrm{A},\left\{v^{k}\right\}$ is uniformly bounded by $M:=\|u\|\left\|g_{\eta}\right\|$. Moreover, $v^{k}$ converges pointwise to $v$ because, by Hypothesis B and Scheffé's Theorem (see, for instance, pp. 223-224 in [2])

$$
\begin{gathered}
\left|v^{k}(x)-v(x)\right| \leq 2 M \int_{\mathrm{X}}\left|g_{\xi}\left(x^{\prime}-F\left(x, a^{k}\right)\right)-g_{\xi}\left(x^{\prime}-F(x, a)\right)\right| \lambda_{1}\left(d x^{\prime}\right) \\
\rightarrow 0 \quad \text { as } k \rightarrow \infty .
\end{gathered}
$$

A similar argument shows that each $v^{k}$ is continuous, and, therefore, $\left\{v^{k}\right\}$ satisfies the conditions (a) and (b) in Lemma 3.2. Finally, observe from (2.27), (2.28) and (2.29) that, as $\varphi^{k} \rightarrow \varphi$ weakly, Lemma 3.2 yields

$$
\begin{aligned}
\int_{\mathrm{X}} u(x) \sigma\left(\varphi^{k}, a^{k}, y^{k}\right)(d x) & =\int_{\mathrm{X}} v^{k}(x) \varphi^{k}(d x) \\
& \rightarrow \int_{\mathrm{X}} v(x) \varphi(d x)=\int_{\mathrm{X}} u(x) \sigma(\varphi, a, y)(d x) .
\end{aligned}
$$

This fact and (2.26) imply that

$$
H\left(\varphi^{k}, a^{k}, y^{k}\right) \rightarrow H(\varphi, a, y) \text { weakly, }
$$

and Assumption 2.3(a) follows.

The limiting PO system. For each $n \in \mathbb{N}_{\infty}$, consider the PO control system

$$
x_{t+1}=F_{n}\left(x_{t}, a_{t}\right)+\xi_{t}, \quad y_{t}=G_{n}\left(x_{t}\right)+\eta_{t},
$$

where $F_{n}(x, a)$ and $G_{n}(x)$ are functions that satisfy (1.3). For $n=\infty$, we have the limiting PO system (1.4). We will use a subindex " $n$ " to indicate functions and probabilities corresponding to the model in (2.31). For instance, the $\alpha$-discounted cost and the optimal cost function in (2.2) and (2.3) become

$$
V_{n}(\pi, \varphi):=\sum_{t=0}^{\infty} \alpha^{t} E_{n, \varphi}^{\pi}\left[c\left(x_{t}, a_{t}\right)\right]
$$

and

$$
V_{n}^{*}(\varphi):=\inf _{\pi} V_{n}(\pi, \varphi)
$$

respectively. 
Theorem 2.6. Suppose that for each finite $n \in \mathbb{N}$, (2.31) satisfies Assumptions

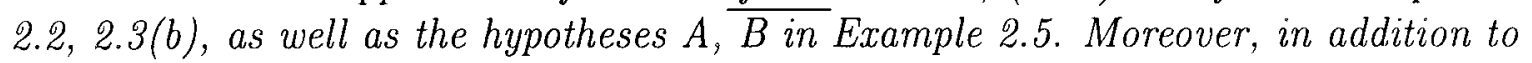
(1.3) we suppose that the limiting functions $F_{\infty}(x, a)$ and $G_{\infty}(x)$ are continuous, and also that for each pair $(\varphi, a)$ in $\mathbb{P}(\mathrm{X}) \times A$, there exists a finite measure $\gamma \equiv \gamma_{\varphi, a}$ on $\mathcal{B}(\mathbb{P}(\mathrm{X}))$ such that

$$
\widehat{P}_{n}(\cdot \mid \varphi, a) \leq \gamma(\cdot) \quad \forall n \in \mathbb{N} .
$$

Then Theorem 2.4 holds for $n=\infty$. Further, if the cost-per-stage $c(x, a)$ is a continuous bounded function, then the condition (2.32) can be omitted.

Theorem 2.6 is proved in $\S 4$.

\section{Proof of Theorem 2.4}

Theorem 2.4 will follow from the results in $\S 8.5$ of [9] if we show that the CO model (2.7)-(2.9) satisfies the Assumptions 8.3.2, 8.3.3 and 8.5.1 in [9]. (As we are assuming that $c(x, a)$ is nonnegative, the continuity condition 8.5.3 in $[9$, p.66] is not required, and, moreover, the continuity of $\widehat{w}(\varphi)$ in condition 8.5 .2 can be replaced with lower semicontinuity.) Thus in view of our current Assumptions 2.2 and 2.3, we only need to verify:

(i) The function $\widehat{w}(\varphi):=\int_{\mathrm{X}} w(x) \varphi(d x)$ is l.s.c. on $\mathbb{P}(\mathrm{X})$.

(ii) The function $\widehat{c}(\varphi, a)$ in (2.8) is l.s.c. on $\mathbb{P}(\mathrm{X}) \times A$.

(iii) The transition law $\widehat{P}(\cdot \mid \varphi, a)$ in $(2.17)$ is weakly continuous, that is, for each $u$ in $C_{b}(\mathbb{P}(\mathrm{X}))$, the function

$$
\widehat{u}(\varphi, a):=\int_{\mathbb{P}(\mathrm{X})} u\left(\varphi^{\prime}\right) \widehat{P}\left(d \varphi^{\prime} \mid \varphi, a\right)
$$

is continuous in $(\varphi, a) \in \mathbb{P}(\mathrm{X}) \times A$.

Parts $(i)$ and $(i i)$ are consequence of the following general result.

Lemma 3.1. Let $\mathrm{X}$ be an arbitrary Borel space. Suppose that $\left\{\varphi^{n}\right\}$ is a sequence in $\mathbb{P}(\mathrm{X})$ converging weakly to $\varphi$, and let $\left\{v^{n}\right\}$ be a sequence of nonnegative and l.s.c. functions on $\mathrm{X}$ such that

$$
\liminf _{n \rightarrow \infty} v^{n}(x) \geq v(x) \quad \forall x \in \mathrm{X}
$$


Then

$$
\liminf _{n \rightarrow \infty} \int_{\mathrm{X}} v^{n}(x) \varphi^{n}(d x) \geq \int_{\mathrm{X}} v(x) \varphi(d x)
$$

Proof. As each $v^{n}$ is nonnegative and l.s.c., for each $n$ there is a sequence $\left\{u_{n, k}\right\}$ in $C_{b}(\mathrm{X})$ such that $u_{n, k} \uparrow v^{n}$ as $k \rightarrow \infty$. Therefore, for all $n, k$, and $m \leq n$

$$
\begin{aligned}
\int_{\mathrm{X}} v^{n}(x) \varphi^{n}(d x) & \geq \int_{\mathrm{X}} u_{n, k}(x) \varphi^{n}(d x) \\
& \geq \int_{\mathrm{X}} \inf _{j \geq m} u_{j, k}(x) \varphi^{n}(d x)
\end{aligned}
$$

Hence, as $\inf _{j \geq m} u_{j, k}(\cdot)$ is in $C_{b}(X)$ and $\varphi^{n} \rightarrow \varphi$ weakly, we get

$$
\liminf _{n \rightarrow \infty} \int_{\mathrm{X}} v^{n}(x) \varphi^{n}(d x) \geq \int_{\mathrm{X}} \inf _{j \geq m} u_{j, k}(x) \varphi(d x) .
$$

Finally, letting $m \rightarrow \infty$, and then $k \rightarrow \infty$, monotone convergence yields

$$
\liminf _{n \rightarrow \infty} \int_{X} v^{n}(x) \varphi^{n}(d x) \geq \int_{X} \liminf _{n \rightarrow \infty} v^{n}(x) \varphi(d x)
$$

and so (3.2) follows from (3.1).

In Lemma 3.1, the case $v^{n}(\cdot) \equiv v(\cdot)$ is well-known; see, for instance, statement (12.3.37) in p.225 of [9]. In particular, we get:

Proof of $(i)$. Take $v^{n}(\cdot) \equiv w(\cdot)$ in Lemma 3.1

We also have the following.

Proof of $(i i)$. Let $\left(\varphi^{n}, a^{n}\right)$ be a sequence in $\mathbb{P}(\mathrm{X}) \times A$ that converges to $(\varphi, a)$. We wish to show that

$$
\liminf _{n \rightarrow \infty} \widehat{c}\left(\varphi_{n}, a_{n}\right) \geq \widehat{c}(\varphi, a)
$$

that is, by (2.8),

$$
\liminf _{n \rightarrow \infty} \int_{\mathrm{X}} c\left(x, a^{n}\right) \varphi^{n}(d x) \geq \int_{\mathrm{X}} c(x, a) \varphi(d x) .
$$

This, however, follows from Lemma 3.1 with $v^{n}(x):=c\left(x, a^{n}\right)$ and $v(x):=c(x, a)$.

To prove (iii) we first note the following general fact. 
Lemma 3.2. Let $\mathrm{X}$ be an arbitrary Borel space, and let $\left\{u_{n}\right\}$ and $\left\{\mu_{n}\right\}$ be sequences in $C_{b}(\mathrm{X})$ and $\mathbb{P}(\mathrm{X})$, respectively, such that:

(a) $\left\{u_{n}\right\}$ is uniformly bounded, that is, $\left\|u_{n}\right\| \leq M$ for some constant $M$;

(b) $u_{n} \rightarrow u$ pointwise; and

(c) $\mu_{n} \rightarrow \mu$ weakly.

Then

$$
\lim _{n \rightarrow \infty} \int_{\mathrm{X}} u_{n} d \mu_{n}=\int_{\mathrm{X}} u d \mu
$$

Proof. By (a) and (b), the nonnegative sequence $v^{n}:=u_{n}+M$ satisfies the hypotheses of Lemma 3.1. Thus, by (c) and (3.2), we obtain

$$
\liminf _{n \rightarrow \infty} \int_{\mathrm{X}} u_{n} d \mu_{n} \geq \int_{\mathrm{X}} u d \mu
$$

Finally, applying the latter inequality to $-u_{n}$ we get

$$
\limsup _{n \rightarrow \infty} \int_{X} u_{n} d \mu_{n} \leq \int_{X} u d \mu
$$

and (3.3) follows.

We next use Lemma 3.3 to show that the stochastic kernels in $(2.10)-(2.18)$ are all continuous.

Lemma 3.3. Under Assumption 2.2(d), the stochastic kernels $P(\cdot \mid x, a), Q(\cdot \mid x)$, and $\widehat{q}(\cdot \mid \varphi, a)$ are weakly continuous. Hence, under the additional Assumption 2.3(a), $\widehat{P}(\cdot \mid \varphi, a)$ is also weakly continuous, that is, (iii) holds.

Proof. If $u$ is in $C_{b}(\mathrm{X})$, it follows from (2.12) that

$$
\int_{\mathrm{X}} u\left(x^{\prime}\right) P\left(d x^{\prime} \mid x, a\right)=\int_{S} u[F(x, a, s)] \mu(d s)
$$

which, by bounded convergence, is continuous in $(x, a)$. Similarly, if $v$ is in $C_{b}(\mathrm{Y})$, it follows from (2.13) that

$$
\int_{\mathrm{Y}} v(y) Q(d y \mid x)=\int_{S^{\prime}} v\left[G\left(x, s^{\prime}\right)\right] \nu\left(d s^{\prime}\right)
$$


is continuous in $x$. Moreover, from (2.15), taking again $v$ in $C_{b}(\mathrm{Y})$,

$$
\begin{aligned}
\int_{\mathrm{Y}} v(y) \widehat{q}(d y \mid \varphi, a) & =\int_{\mathrm{X}} \int_{\mathrm{X}} \int_{\mathrm{Y}} v(y) Q\left(d y \mid x^{\prime}\right) P\left(d x^{\prime} \mid x, a\right) \varphi(d x) \\
& =\int_{\mathrm{X}} v^{\prime}(x, a) \varphi(d x),
\end{aligned}
$$

where

$$
v^{\prime}(x, a):=\int_{\mathrm{X}} \int_{\mathrm{Y}} v(y) Q\left(d y \mid x^{\prime}\right) P\left(d x^{\prime} \mid x, a\right)
$$

is a continuous function on $\mathrm{X} \times A$, bounded by $\|v\|$. Now let $\left(\varphi^{n}, a^{n}\right) \rightarrow(\varphi, a)$. Then, applying Lemma 3.2 to $u_{n}(\cdot):=v^{\prime}\left(\cdot, a^{n}\right)$ and $\mu_{n}=\varphi^{n}$, we conclude that

$$
\widehat{q}\left(\cdot \mid \varphi^{n}, a^{n}\right) \rightarrow \widehat{q}(\cdot \mid \varphi, a) \quad \text { weakly. }
$$

Finally, if $u$ is in $C_{b}(\mathbb{P}(\mathrm{X})),(2.18)$ gives

$$
\int_{\mathbb{P}(\mathrm{X})} u\left(\varphi^{\prime}\right) \widehat{P}\left(d \varphi^{\prime} \mid \varphi, a\right)=\int_{\mathrm{Y}} u[H(\varphi, a, y)] \widehat{q}(d y \mid \varphi, a),
$$

and so the weak continuity of $\widehat{P}$ follows from (3.4), Assumption 2.3(a) and Lemma 3.2 .

To summarize, the conditions $(i),(i i),(i i i)$ at the beginning of this section yield Theorem 2.4 .

\section{Proof of Theorem 2.6}

For each finite $n \in \mathbb{N}$, the Bellman equation (2.20) becomes

$$
V_{n}^{*}(\varphi)=\min _{a \in A}\left[\widehat{c}(\varphi, a)+\alpha \int_{\mathbf{P}(\mathrm{X})} V_{n}^{*}\left(\varphi^{\prime}\right) \widehat{P}_{n}\left(d \varphi^{\prime} \mid \varphi, a\right)\right]
$$

To verify Theorem 2.4 for $n=\infty$ it suffices to show that $V_{\infty}^{*}$ satisfies (4.1), i.e.,

$$
V_{\infty}^{*}(\varphi)=\min _{a \in A}\left[\widehat{c}(\varphi, a)+\alpha \int_{\mathbb{P}(\mathrm{X})} V_{\infty}^{*}\left(\varphi^{\prime}\right) \widehat{P}_{\infty}\left(d \varphi^{\prime} \mid \varphi, a\right)\right]
$$


because then the uniqueness of solutions to (4.2) in $\mathbb{B}_{w}(\mathbb{P}(\mathrm{X}))$, as well as parts (b) and (c) in Theorem 2.4, follow from Theorem 8.3.6 and statement (8.5.3) in [9].

Now, to prove (4.2), let

$$
\underline{u}(\varphi):=\liminf _{n \rightarrow \infty} V_{n}^{*}(\varphi), \quad \text { and } \quad \bar{u}(\varphi):=\limsup _{n \rightarrow \infty} V_{n}^{*}(\varphi)
$$

We wish to show that

$$
\underline{u}(\varphi)=\bar{u}(\varphi)=V_{\infty}^{*}(\varphi) \quad \forall \varphi \in \mathbb{P}(\mathrm{X})
$$

To prove this, let us first note the following.

Lemma 4.1. As $n \rightarrow \infty$,

(a) $\left\|\widehat{q}_{n}(\cdot \mid \varphi, a)-\widehat{q}_{\infty}(\cdot \mid \varphi, a)\right\|_{T V} \rightarrow 0$ for each $(\varphi, a)$ in $\mathbb{P}(\mathrm{X}) \times A$, where $\|\cdot\|_{T V}$ denotes the total variation norm.

(b) $\left\|H_{n}(\varphi, a, y)(\cdot) \rightarrow H_{\infty}(\varphi, a, y)(\cdot)\right\|_{T \mathrm{~V}} \rightarrow 0$ for all $(\varphi, a, y)$ in $\mathbb{P}(\mathrm{X}) \times A \times \mathrm{Y}$, where $H_{n}$ is the filtering function in (2.26), (2.27).

(c) $\widehat{P}_{n}(\cdot \mid \varphi, a) \rightarrow \widehat{P}_{\infty}(\cdot \mid \varphi, a)$ weakly for each $(\varphi, a)$.

Proof. (a) For each $n \in \mathbb{N}_{\infty}$, let $P_{n}(\cdot \mid x, a)$ and $Q_{n}(\cdot \mid x)$ be as in (2.24) and (2.25), that is,

$$
P_{n}(B \mid x, a)=\int_{B} g_{\xi}\left(s-F_{n}(x, a)\right) \lambda_{1}(d s)
$$

and

$$
Q_{n}(C \mid x)=\int_{C} g_{\eta}\left(s^{\prime}-G_{n}(x)\right) \lambda_{2}\left(d s^{\prime}\right) .
$$

As $g_{\xi}\left(s-F_{n}(x, a)\right) \rightarrow g_{\xi}\left(s-F_{\infty}(x, a)\right)$ for all $(x, a, s)$, it follows from Scheffé's Theorem (see, for instance, pp. 223-224 in [2]) that

$$
\left\|P_{n}(\cdot \mid x, a)-P_{\infty}(\cdot \mid x, a)\right\|_{T V} \rightarrow 0 \quad \forall(x, a) \in \mathrm{X} \times A .
$$

Similarly, as $g_{\eta}\left(s^{\prime}-G_{n}(x)\right) \rightarrow g_{\eta}\left(s^{\prime}-G_{\infty}(x)\right)$, we have

$$
\left\|Q_{n}(\cdot \mid x)-Q_{\infty}(\cdot \mid x)\right\|_{T V} \rightarrow 0 \quad \forall x \in \mathrm{X} .
$$


Therefore, by (2.15), i.e.,

$$
\widehat{q}_{n}(\cdot \mid \varphi, a)=\int_{\mathrm{X}} \int_{\mathrm{X}} Q_{n}\left(\cdot \mid x^{\prime}\right) P_{n}\left(d x^{\prime} \mid x, a\right) \varphi(d x),
$$

a straightforward calculation using (4.4) and (4.5) yields (a).

(b) By (2.26) and (2.27), to prove (b) it suffices to show that, for all $(\varphi, a, y)$,

$$
\sigma_{n}(\varphi, a, y)(B)=\int_{\mathrm{X}}\left[\int_{B} g_{\eta}\left(y-G_{n}\left(x^{\prime}\right)\right) P_{n}\left(d x^{\prime} \mid x, a\right)\right] \varphi(d x)
$$

converges to $\sigma_{\infty}(\varphi, a, y)(B)$ in the total variation norm. To do this observe that, for all $B \in \mathcal{B}(\mathrm{X})$,

$$
\begin{gathered}
\left|\int_{B} g_{\eta}\left(y-G_{n}\left(x^{\prime}\right)\right) P_{n}\left(d x^{\prime} \mid x, a\right)-\int_{B} g_{\eta}\left(y-G_{\infty}\left(x^{\prime}\right)\right) P_{\infty}\left(d x^{\prime} \mid x, a\right)\right| \\
\leq\left\|g_{\eta}\right\|\left\|P_{n}(\cdot \mid x, a)-P_{\infty}(\cdot \mid x, a)\right\|_{T V}+\int_{X}\left|g_{\eta}\left(y-G_{n}\left(x^{\prime}\right)\right)-g_{\eta}\left(y-G_{\infty}\left(x^{\prime}\right)\right)\right| P_{\infty}\left(d x^{\prime} \mid x, a\right) \\
\rightarrow 0 \text { as } n \rightarrow \infty,
\end{gathered}
$$

and the latter convergence is, of course, uniform in $B \in \mathcal{B}(\mathrm{X})$. This clearly implies

$$
\left\|\sigma_{n}(\varphi, a, y)(\cdot)-\sigma_{\infty}(\varphi, a, y)(\cdot)\right\|_{T V} \rightarrow 0 \text { as } n \rightarrow \infty
$$

and (b) follows.

(c) Choose an arbitrary function $u$ in $C_{b}(\mathbb{P}(\mathrm{X}))$. Then, by (2.18),

$$
\int_{\mathbb{P}(\mathrm{X})} u\left(\varphi^{\prime}\right) \widehat{P}_{n}\left(d \varphi^{\prime} \mid \varphi, a\right)=\int_{\mathrm{Y}} u\left[H_{n}(\varphi, a, y)\right] \widehat{q}_{n}(d y \mid \varphi, a) .
$$

Now observe that the integrand $u\left[H_{n}(\varphi, a, y)\right]$ is bounded by $\|u\|$ for all $n$. On the other hand, (a) and (b) imply the weak convergence of $\widehat{q}_{n}(\cdot \mid \varphi, a)$ to $\widehat{q}_{\infty}(\cdot \mid \varphi, a)$, and of $H_{n}(\varphi, a, y)(\cdot)$ to $H_{\infty}(\varphi, a, y)(\cdot)$. Thus (c) follows from (4.6) and Lemma 3.2.

We now go back to the proof of (4.3). First take the lim inf in both sides of (4.1). Then, by Lemma 4.1(c) and Lemma 3.1, we obtain

$$
\underline{u}(\varphi) \geq \min _{a \in A}\left[\widehat{c}(\varphi, a)+\alpha \int_{\mathbf{P}(\mathrm{X})} \underline{u}\left(\varphi^{\prime}\right) \widehat{P}_{\infty}\left(d \varphi^{\prime} \mid x, a\right)\right] .
$$


Therefore, by a standard dynamic programming argument (see, for instance, Lemma 4.2 .7 in [8])

$$
\underline{u}(\varphi) \geq V_{\infty}^{*}(\varphi) \quad \forall \varphi \in \mathbb{P}(\mathrm{X})
$$

To complete the proof of $(4.3)$, we next show that

$$
\bar{u}(\varphi) \leq V_{\infty}^{*}(\varphi) \quad \forall \varphi \in \mathbb{P}(X)
$$

which together with (4.8) yields (4.3). To obtain (4.9) we see from (4.1) that

$$
V_{n}^{*}(\varphi) \leq \widehat{c}(\varphi, a)+\alpha \int_{\mathbb{P}(\mathrm{X})} V_{n}^{*}\left(\varphi^{\prime}\right) \widehat{P}_{n}\left(d \varphi^{\prime} \mid \varphi, a\right)
$$

for all $(\varphi, a)$ in $\mathbb{P}(\mathrm{X}) \times A$. Furthermore, by the hypothesis (2.32) and Lemma 4.1(c), $\widehat{P}_{n}(\cdot \mid \varphi, a)$ converges setwise to $\widehat{P}_{\infty}(\cdot \mid \varphi, a)$ for each $(\varphi, a)$; see, for instance, Lemma $4.1(i i)$ in [15]. In addition, the sequence $V_{n}^{*}(\varphi)$ is uniformly bounded by $C \widehat{w}(\varphi) /(1-$ $\alpha \beta$ ), where $C$ and $\beta$ are the constants in Assumptions 2.2(f) and 2.3(b), respectively; see p.52, inequality (8.3.33), in [9]. It follows that the Extended Fatou Lemma 8.3.7(b) in [9] is applicable to (4.10), so that taking the lim sup as $n \rightarrow \infty$ we get

$$
\bar{u}(\varphi) \leq \widehat{c}(\varphi, a)+\alpha \int_{\mathbb{P}(\mathrm{X})} \bar{u}\left(\varphi^{\prime}\right) \widehat{P}_{\infty}\left(d \varphi^{\prime} \mid \varphi, a\right)
$$

This implies that

$$
\bar{u}(\varphi) \leq \min _{a \in A}\left[\widehat{c}(\varphi, a)+\alpha \int_{\mathbb{P}(\mathrm{X})} \bar{u}\left(\varphi^{\prime}\right) \widehat{P}_{\infty}\left(d \varphi^{\prime} \mid \varphi, a\right)\right],
$$

which in turn, by Lemma 4.2.7 in [8], for instance, yields (4.9).

This completes the proof of (4.3), and hence of (4.2), when the cost-per-stage function $c$ satisfies Assumption 2.2(e), (f). Finally, if $c$ is continuous and bounded on $\mathrm{X} \times A$, it follows from Lemma 3.3, together with Theorem 2.8 in [7, p.23], that $\left\{V_{n}^{*}, n \in \mathbb{N}\right\}$ is a uniformly bounded sequence of continuous functions on $\mathbb{P}(\mathrm{X})$. Hence, from Lemma 3.1 with an obvious change, we can obtain (4.11) directly from (4.10), without using (2.32). 


\section{Concluding remarks}

As was already mentioned, the results in Theorem 2.4 are essentially well known except for the fact that $c(x, a)$ is allowed to be unbounded and for the generality of the PO system (1.5). However, to our knowledge, the proof itself is new. In fact, even the Lemmas 3.1 and 3.2 are new. Similarly, parts (a) and (b) in Lemma 4.1, which concern the total variation norm, seem to be new.

In fact, observe that Lemma 3.1 is a significant extension of the standard Fatou's Lemma, namely,

$$
\liminf _{n \rightarrow \infty} \int_{\mathrm{X}} v^{n}(x) \varphi(d x) \geq \int_{\mathrm{X}}\left[\liminf _{n \rightarrow \infty} v^{n}(x)\right] \varphi(d x)
$$

in which $v^{n}$ is a sequence of nonnegative measurable functions, as well as an extension of the Extended Fatou Lemma 8.3.7 in [9], in which (3.2) holds for a sequence of probability measures $\varphi^{n}$ converging setwise to $\varphi$. Similarly, Lemma 3.2 is an extension of the standard Bounded Convergence Theorem, in which the measures $\mu_{n} \equiv \mu$ are fixed.

On the other hand, Theorem 2.4 includes the important case in which the state space $\mathrm{X}$ and the observation set $\mathrm{Y}$ are countable, as occurs in many applications $[3,5$, $6,13,16,17,20, \cdots]$. In such a case, the filtering function $H$ turns out to be similar to $(2.26)$, with

$$
\sigma(\varphi, a, y)\left(x^{\prime}\right)=Q\left(y \mid x^{\prime}\right) \sum_{x} P\left(x^{\prime} \mid x, a\right) \varphi(x)
$$

(compare with (2.27)), and so Assumptions 2.2 and 2.3 can be simplified in the obvious manner.

\section{References}

[1] D.P. Bertsekas and S.E. Shreve, Stochastic Optimal Control: The Discrete Time Case, Academic Press, New York, 1978.

[2] P. Billingsley, Convergence of Probability Measures, Wiley, New York, 1968.

[3] T.E. Duncan, B. Pasik-Duncan and L. Stettner, "Adaptive control of a partially observed discrete time Markov process", Appl. Math. Optim. 37 (1998), 269-293. 
[4] P.K. Dutta, M.K. Majumdar and R.K. Sundaram, "Parametric continuity in dynamic programming models", J. Econ. Dyn. Control 18 (1994), 1069-1092.

[5] R.J. Elliott, L. Aggoun and J.B. Moore, Hidden Markov Models: Estimation and Control, Springer-Verlag, New York, 1994.

[6] E. Fernández-Gaucherand, A. Arapostathis and S.I. Marcus, "Analysis of an adaptive control scheme for a partially observed Markov chain", IEEE Trans. Autom. Control 38 (1993), 987-993.

[7] O. Hernández-Lerma, Adaptive Markov Control Processes, Springer-Verlag, New York, 1989.

[8] O. Hernández-Lerma and J.B. Lasserre, Discrete-Time Markov Control Processes: Basic Optimality Criteria, Springer-Verlag, New York, 1996.

[9] O. Hernández-Lerma and J.B. Lasserre, Further Topics on Discrete-Time Markov Control Processes, Springer-Verlag, New York, 1999.

[10] O. Hernández-Lerma and S.I. Marcus, "Adaptive control of Markov processes with incomplete state information and unknown parameters", J. Optim. Theory Appl. 52 (1987), 227-241.

[11] O. Hernández-Lerma and S.I. Marcus, "Nonparametric adaptive control of discrete-time partially observable stochastic systems", J. Math. Anal. Appl. 137 (1989), 312-334.

[12] N. Hilgert and O. Hernández-Lerma, "Limiting optimal discounted-cost control of a class of time-varying stochastic systems." Syst. Control Lett. (To appear.)

[13] D.E. Lane, "A partially observable model of decision making by fishermen", Oper. Res. 37 (1989), 240-254.

[14] H.-J. Langen, "Convergence of dynamic programming models", Math. Oper. Res. 6 (1981), 493-512.

[15] J.B. Lasserre, "On the setwise convergence of sequences of measures", J. Appl. Math. Stoch. Anal. 10 (1997), 131-136.

[16] J.A. Loewe, Markov Decision Chains with Partial Information, Ph.D. Thesis, Department of Mathematics and Computer Science, Leiden University, 1995. 
[17] G.E. Monahan, "A survey of partially observable Markov decision processes: theory, models, and algorithms", Manage. Sci. 28 (1982), 1-16.

[18] A. Müller, "How does the value function of a Markov decision process depend on the transition probability?", Math. Oper. Res. 22 (1997), 872-885.

[19] K.R. Parthasarathy, Probability Measures on Metric Spaces, Academic Press, New York, 1971.

[20] W.J. Runggaldier and L. Stettner, Approximations of Discrete Time Partially Observed Control Problems, Applied Mathematics Monographs CNR 6, Giardini, Pisa, 1994

[21] C. Striebel, Optimal Control of Discrete Time Stochastic Systems, Lecture Notes Econ. Math. Syst. 110, Springer-Verlag, Berlin, 1975.

[22] A.A Yushkevich, "Reduction of a controlled Markov model with incomplete data to a problem with complete information in the case of Borel state and control spaces", Theory Probab. Appl. 21 (1976), 153-158. 


\section{WORKING PAPERS 1999}

\section{Business Economics Series}

99-01 (01) Jaime Ortega

"Power in the firm and managerial career concerns"

99-06 (02) Sandro Brusco

"Short-termism as optimal experimentation policy"

99-07 (03) A. Balbás, I.R. Longarela y J. Lucia

"How Does Financial Theory Apply to Catastrophe-Linked Derivatives?

An Empirical Test of Several Pricing Models"

99-17 (04) Ashish Arora, Andrea Fosfuri and Alfonso Gambardella

"Markets for technology (why do we see them, why don't we see more of them, and why we should care"

99-18 (05) María José Álvarez and Jaime Rivera

"An integrated typology of green manufacturing profiles"

99-30 (06) Sandro Brusco and Giuseppe Lopomo

"Collusion via Signalling in Open Ascending Auctions with Multiple Objects and Complementarities"

99-33 (07) Margarita Samartín

"Costly Financial Crises"

99-35 (08) Ángel Pardo, Alejandro Balbás and Vicente Meneu

"On the effectiveness of several market integration measures. An empirical analysis"

99-39 (09) Ester Martínez-Ros and Josep A. Tribó

"R\&D and Debt Financing"

99-40 (10) Carolina Manzano

"Integratin versus segmentation in a dealer market"

99-41 (11) Elizabeth F. Cabrera and Ángel Cabrera

"The State of Strategic Human Resource Measurement in Spanish Banks"

99-46 (12) Ángel Cabrera, Elizabeth F. Cabrera and Sebastian Barajas

"Organizational culture as a determinant of technology assimilation"

99-47 (13) Elizabeth F. Cabrera and Ángel Cabrera

"Rethinking utility analysis: A strategic focus"

99-61 (14) Carlos Larrinaga, Francisco Carrasco, Carmen Correa, Francisco Javier Caro and José María Páez.

"The Role of Environmental Accounting in Organizational Change: An Exploration of Spanish Companies" 